\title{
Optimal planning of selective waste collection
}

\author{
S. Raicu, D. Costescu, E. Roşca \& M. Popa \\ University Politehnica of Bucharest, Romania
}

\begin{abstract}
The paper presents an optimisation method for selective waste collection services in university campuses in Bucharest. Based on geographic information system (GIS) procedures and algorithms, an optimisation method for selective waste collection services is developed. The area of Bucharest City, the road network, the location of the selective waste collection points in the main university campuses and the location of the collection station are organized in GIS format. Complex geodatabase structures that allow solving the large scale selective waste collection problem are designed. The routing solution that minimises the total travel time is determined taking into consideration the local road network properties and the location of the collection station for each category of waste (glass, paper and plastic). The methodology is applied for the main University Campuses in Bucharest. The map with the routing solution, the required number of vehicles and the schedule for each category of waste and for each vehicle are obtained. The developed method provides informational and technological support for public authorities and selective waste collection operators.

Keywords: selective waste collection, optimisation, vehicle routing, scheduling, geographic information system.
\end{abstract}

\section{Introduction}

The problem presented in this paper is from a study carried out for the selective waste collection management system in university campuses in Bucharest. With over 250000 students, Bucharest is the greatest national university centre of Romania. There are over 15 important universities with academic, administrative and residential activities organized in campuses spread out in different areas of the city. The campuses have different organization systems, according with the structure, location, tradition and evolution of each university. Due to the large 
number of the student and employees in all these campuses, selective waste management is essential for minimizing the impact on the environment.

Since 2006 the municipal authority in partnership with waste collection cooperatives and associations has been developing a selective waste collection management system. For the period February - July 2006 a pilot project was applied for selective collection of paper, glass and paper in the area of University Politehnica of Bucharest. In that stage were collected about $22000 \mathrm{~kg}$ recyclable wastes, from which $12000 \mathrm{~kg}$ paper, $7000 \mathrm{~kg}$ plastic and $3000 \mathrm{~kg}$ glass. Since then the system has been developed in other campuses. Figure 1 shows the increasing volume of selective waste collected in university campuses.

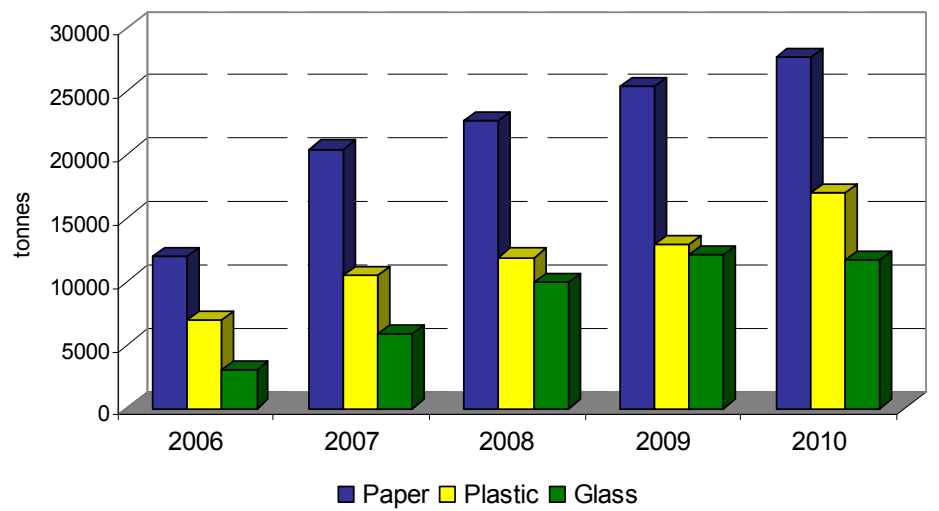

Figure 1: Volume of selective waste collected in university campuses.

In order to increase the efficiency and effectiveness of the selective collection system, optimisation methods are necessary for services planning. In this paper we describe methods for vehicle routing and collection service scheduling. Examples for campuses located in one of the six administrative sectors of the Bucharest are given.

\section{Selective collection model overview}

The optimisation of selective collection services implies solving transport routing and scheduling problems. The objectives of these two combinatorial problems consist of minimising the total haulage time, the fleet size, the transport costs or an aggregated function of the above mentioned.

The problem is defined on a graph $G=(J, D)$, where $J=K \cup L$; $K=\left\{k_{1}, k_{2}, \ldots, k_{N}\right\}$ is the vertex set including the collection waste centres or warehouses for paper, plastic and glass and $L=\left\{l_{1}, l_{2}, \ldots, l_{M}\right\}$ is the vertex set including the selective collection points where blue containers for paper, yellow container for plastic and green container for glass are located. The arc set is 
defined as $D=\left\{\left(k_{i}, l_{j}\right) \mid k_{i} \in K, l_{j} \in L\right\} \cup\left\{\left(l_{i}, l_{j}\right) \mid l_{i}, l_{j} \in L, i \neq j\right\}$. Each arc has associated a non-negative value $t_{i j}$ representing travel time.

Each selective collection point $l_{i}$ has associated a non-negative value $t_{p}$ representing the waste container picking time. Three containers (for selective paper, plastic and glass waste) with the capacity $V_{\text {sel }}=3 \mathrm{~m}^{3}$ are located in each collection point.

On a single route, a vehicle can collect three containers for the same category of waste (Figure 2). Different container filling intensities are registered for each type of waste. Therefore each selective collection point has associated a set of picking periods $\left\{T_{i}^{I} \mid w \in\{1,2,3\}\right\}$, where $w=1$ for paper container, $w=2$ for plastic container and $w=3$ for glass container.

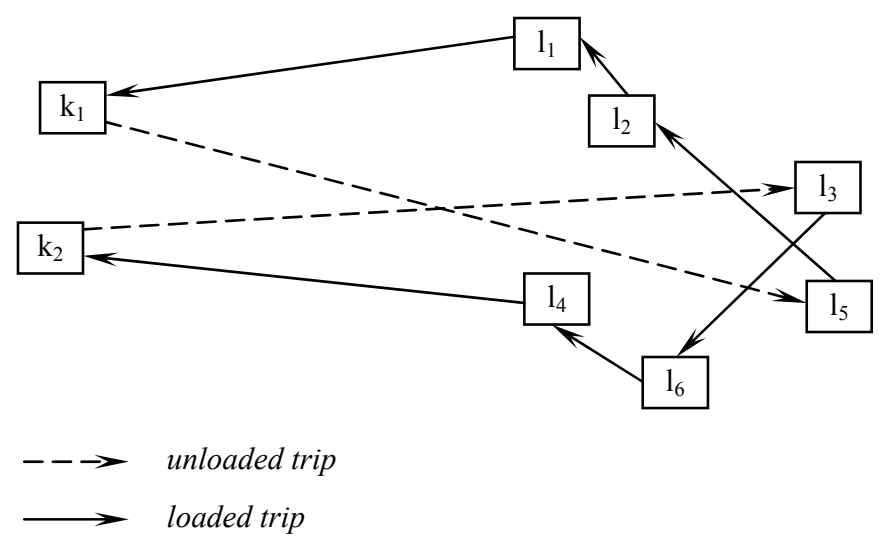

Figure 2: $\quad$ Examples of vehicle trip.

The Vehicle Routing Problem (VRP) involves finding a set of routes, starting and ending at one collection centre, which services a set of selective collection points. The objective function is to minimise the total travel time. Let $Z=\left\{1,2, \ldots, Z_{\max }\right\}$ denote the set of trips that satisfy all the collecting demands for all categories of waste. The routing phase can be mathematically expressed as:

$$
\text { Minimize } \sum_{w=1}^{3} \sum_{z \in Z} \alpha_{w z}\left(\sum_{i=1}^{N} \beta_{w i} \cdot \rho_{z i}\left(\sum_{j=1}^{M} \delta_{z j} \cdot t_{i j}\right)+\sum_{i=1}^{M} \sum_{j=1}^{M} \delta_{z j} \cdot t_{i j}\right)
$$

subject to

$$
\sum_{w=1}^{3} \sum_{z \in Z} \alpha_{w z}=Z_{\max }
$$




$$
\begin{aligned}
& \sum_{w=1}^{3} \sum_{i=1}^{N} \beta_{w i} \leq N \\
& \sum_{z \in Z} \sum_{i=1}^{N} \rho_{z i}=\left|\frac{\max _{i}\left\{T_{i}^{w}\right\}}{\min _{i}\left\{T_{i}^{w}\right\}}\right|, \forall w \in\{1,2,3\} \\
& \sum_{z \in Z} \delta_{z j}=\left|\frac{\max _{i}\left\{T_{i}^{w}\right\}}{T_{j}^{w}}\right|, \forall w \in\{1,2,3\}, \forall j \in\{1,2, \ldots, M\}
\end{aligned}
$$

where

$\alpha_{w z}$ : decision variable; $\alpha_{w z}=1$ if the trip $z$ is planned for the waste $w$; $\alpha_{w z}=0$ otherwise.

$\beta_{w i}$ : decision variable; $\beta_{w i}=1$ if the centre $k_{i}$ collect category $w$ of waste; $\beta_{w i}=0$ otherwise.

$\rho_{z i}$ : decision variable; $\rho_{z i}=1$ if the trip $z$ starts from centre $k_{i}$ and $\rho_{z i}=0$ otherwise.

$\delta_{z j}$ : decision variable; $\delta_{z j}=1$ if the trip $z$ serve the point $l_{j}$ and $\rho_{z j}=0$ otherwise.

The objective function (1) measures the total travel time of the system. Equation (2) expresses the trip allocation on waste category collection. Relation (3) is a constraint related to the type of the collection centres. The restrictions (4) and (5) express the demand satisfaction restrictions.

When for each collection point, a time interval $I=\left[\tau_{\min }^{i}, \tau_{\max }^{i}\right]$ for picking operation is settled according with the location in the campus (faculties and administrative buildings area or student residential area), the problem turns into a Vehicle Routing Problem with Time Windows (VRPTW). Bodin [1] presented an overview of VRPTW optimisation methods. In the case of selective waste collection presented in this paper, a geographic information system (GIS) model is developed for solving VRPTW. Allocation and routing procedure available in ArcGIS - Network Analyst Extension are used. The section 4 provides a description of the logical structure designed for the GIS model.

In order to solve the VRPTW, we have to schedule the collecting trips according with the periodically demands. The problem deals with finding the subsets of collection points, which have to be served at the same time. The next section presents an algorithm for selecting and ordering the sets of selective collection containers, which are to be processed on a task. The algorithm is applied for each waste category and for the given set of picking periods. 
Vehicle routing problem is solved for each waste collection centre and for each set of containers obtained as solution in scheduling algorithm. Finally, the detailed vehicle schedules are calculated, with respect to the time window constraints.

\section{Collection planning algorithm}

Different deterministic scheduling methods are developed for practical application (Martelo et al. [2]). The problem of scheduling containers picking operation needs a peculiar approach. As mentioned before, the sets of picking periods $\left\{T_{i}^{w}\right\}$ are given for container location $l_{i} \in L$ and for each category of selective waste $w \in\{1,2,3\}$. The first step consists in identifying the subsets of collection points with simultaneous demands for a selective waste category (Figure 3). We describe below the algorithm built for solving this issue.

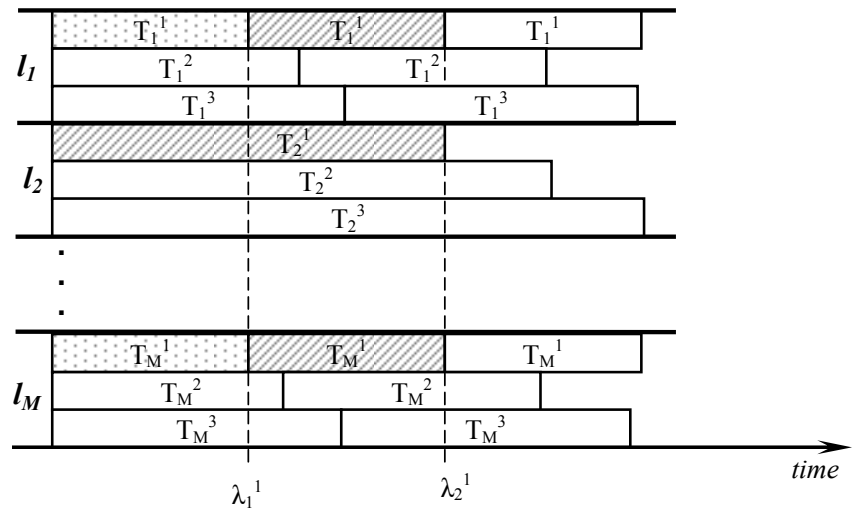

Figure 3: Gantt chart of picking periods.

We consider the following parameters:

- $\quad$ The vector of collecting times $\Gamma^{w}=\left\{\gamma_{1}^{w}, \gamma_{2}^{w}, \ldots, \gamma_{M}^{w}\right\}$ where $w \in\{1,2,3\}$ and $\gamma_{i}^{w}$ is the time when in the location $l_{i}$ the container for the waste $w$ have to be collect.

- The due date parameters $\lambda_{j}^{w}$ representing the times when collection operation has to be processed for the waste $w$.

- The set of tasks $Z_{j}^{w}$ including all location $l_{k}$ which have to be served at the time $\lambda_{j}^{w}$

- $\quad$ The set of scheduling period $\left\{T_{\max }^{w}\right\}, w \in\{1,2,3\}$. 
The algorithm for solving the container allocation on collection tasks follows the next steps:

\section{Parameter initialization}

1.1. Assign the given period to any collection time $\gamma_{i}^{w}=T_{i}^{w}$, $i \in\{1,2, \ldots, M\}$.

1.2. Assign for the first iteration $j=1$ and $\lambda_{j}^{w}=\min _{i \leq M} \lambda_{j}^{w}$ and $Z_{j}^{w}=\Phi$.

1.3. Assign the maximum period to the scheduling period $T_{\max }^{w}=\max _{i \leq M} T_{i}^{w}$.

2. Iteration

2.1. For all $i \in\{1,2, \ldots, M\}$, if $\gamma_{i}^{w}=\lambda_{i}^{w}$ then $\mathrm{Z}_{j}^{w} \leftarrow \mathrm{Z}_{j}^{w} \cup\left\{l_{i}\right\}$, $\lambda_{i}^{w} \leftarrow \lambda_{i}^{w}+T_{i}^{w}$ and go to step 2.2.

2.2. Calculate the parameters for the next iteration $j \leftarrow j+1$, $\lambda_{j}^{w}=\min _{i \leq M} \lambda_{i}^{w}$. Until $\lambda_{j}^{w} \leq T_{\max }^{w}$ go to step 2.1. If $\lambda_{j}^{w}>T_{\max }^{w}$ then go to step 3.

3. Saving the solution

3.1. Export the sets of task $Z_{j}^{w}$ in a table format, which will be used as input data in the GIS model.

\section{Vehicle routing model}

Based on geographic information system (GIS) procedures, a model for solving the vehicle routing problem is developed. The model is applied for identifying the routes starting and ending at one collection centre and serving the set of selective collection containers. The routing solution that minimises the total travel time is determined taking into consideration the local road network properties and the location of the collection containers for each category of waste (glass, paper and plastic).

Before the routing model could be constructed, preparing geospatial databases is necessary. The datasets used in the GIS model include:

- Area of administrative sectors of Bucharest City;

- Road network;

- Area of university campuses;

- Location of the selective waste collection centres;

- Location of the selective waste collection containers in the university campuses.

The map representation of the geospatial datasets is shown in figure 4 . 


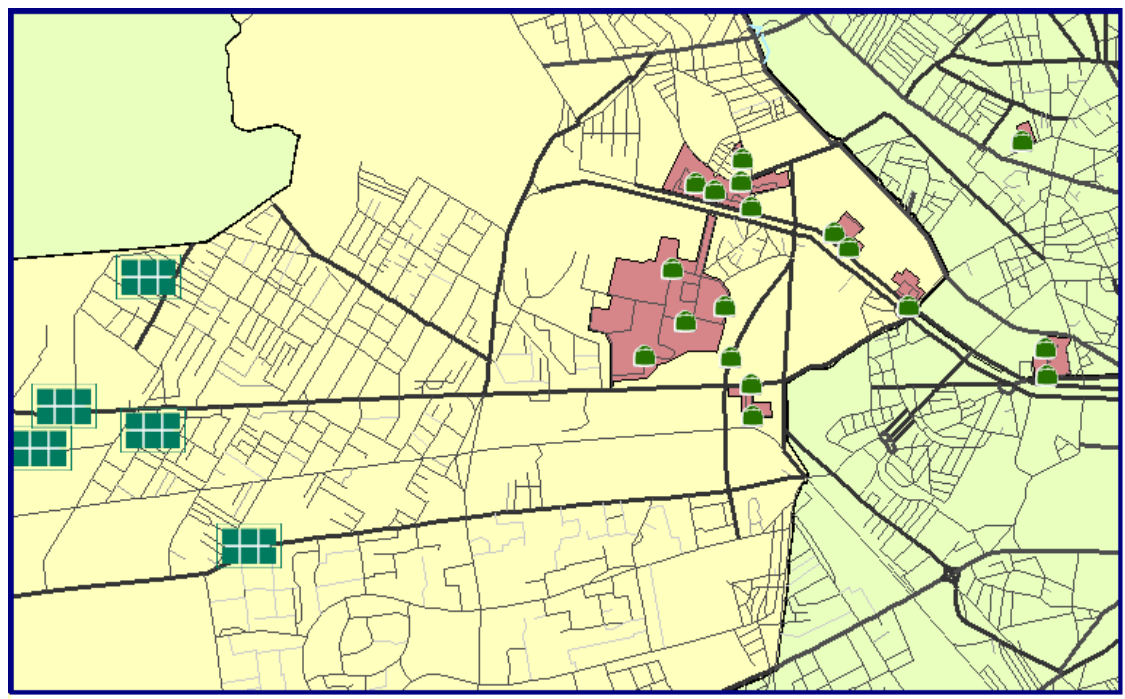

\section{Legend}

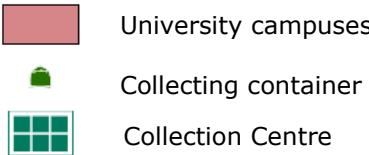

Figure 4: $\quad$ Map of the model features.

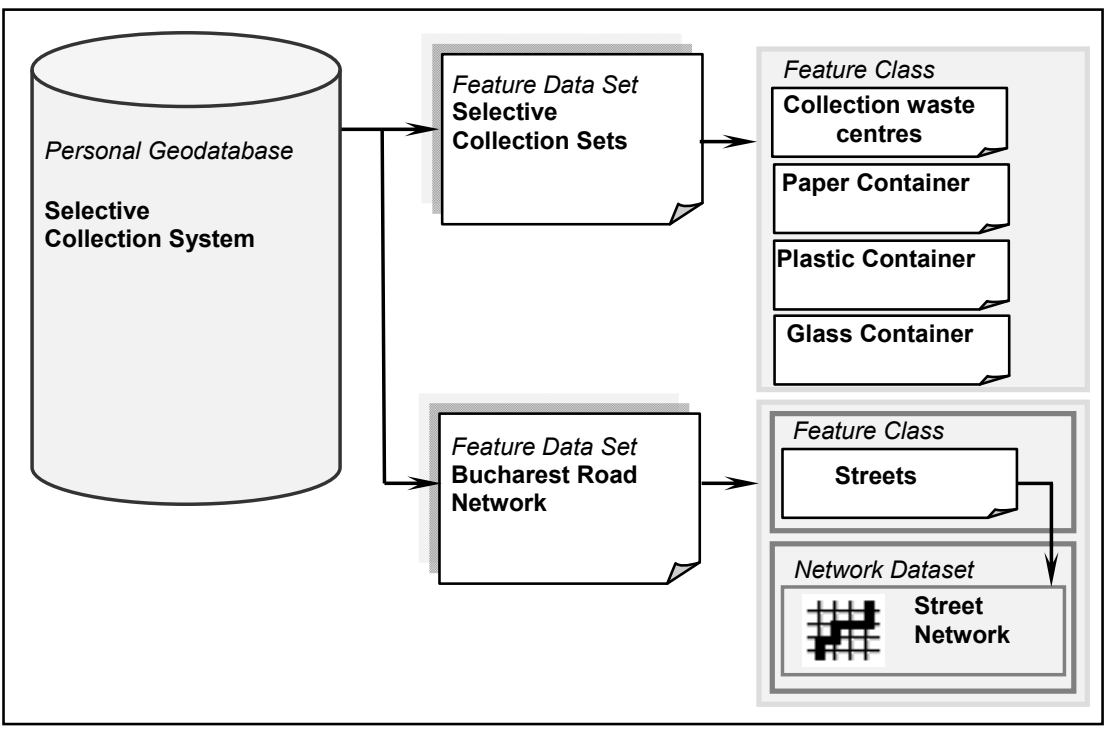

Figure 5: Elements in network datasets creation. 
In order to create a network dataset and solving the large scale selective waste collection problem is necessary to organise all the datasets in an integrated geodatabase structure (Figure 5). A network dataset is created based on the road network geospatial database. Connectivity rules are applied and time and distance attributes are used as evaluators in the network dataset.

With all the geospatial data digitized and necessary attributes added, the vehicle routing model can be built. The ArcToolBox - ArcGIS software is used for creating the model shown in Figure 6. Procedure "Make Route Layer" is applied for the street network dataset. The selective collection containers are located on the route layer and "Network Analyst" procedure is used for solving the vehicle routing problem.

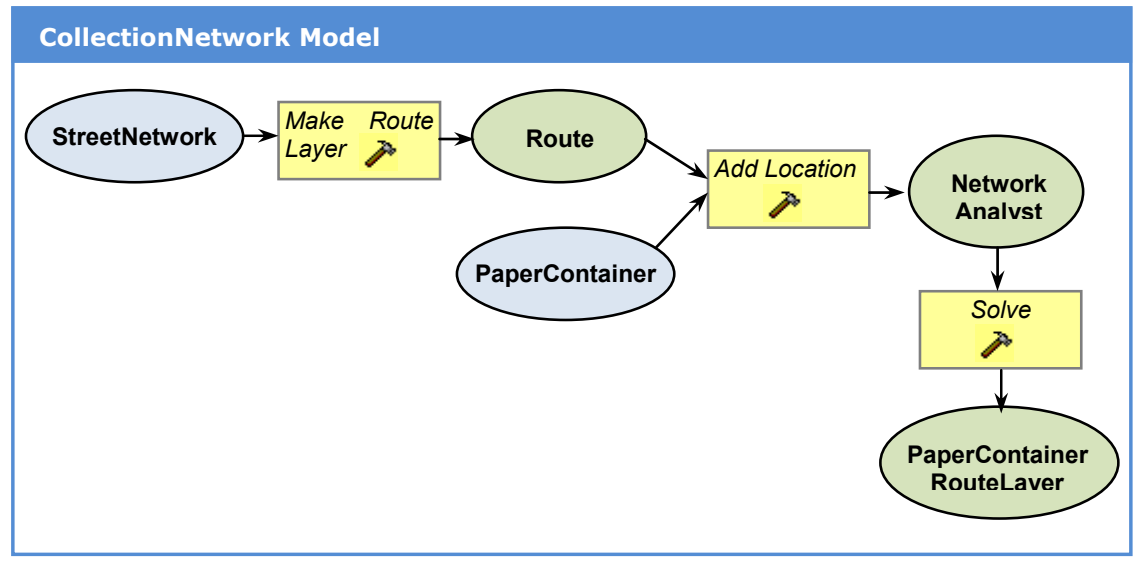

Figure 6: Flow chart of the vehicle routing GIS model.

The model has to be successively applied for each set of selective waste containers (paper, plastic and glass) and for all sets of task $Z_{j}^{I}$ established in the previous step.

\section{Case study}

The model described before has been applied to optimise the selective waste collection system in university campuses located in the Sixth Administrative Sector of Bucharest. Figure 4 shows the location of the selective waste containers and collecting warehouses.

The previous presented algorithm has been used in order to planning the collection services. A collecting tasks selection is presented in Table 1. The routing problem has been solved successively for each task with the developed GIS model. The route layer resulted for the first task is shown in Figure 7. 
Table 1: Collecting services.

\begin{tabular}{|c|c|c|c|c|c|}
\hline OBJECTID & CollectionPoint & W Id & Period & Allocation & Task \\
\hline 1 & Automatica & 1 & 5 & Wahrehouse \#05 & $\# 10501$ \\
\hline 2 & Rectorat & 1 & 5 & Wahrehouse \#05 & $\# 10501$ \\
\hline 3 & Mecanica & 1 & 10 & Wahrehouse \#05 & $\# 10503$ \\
\hline 4 & Transporturi & 1 & 20 & Wahrehouse \#05 & $\# 10506$ \\
\hline 5 & IntrareVasileMilea & 1 & 10 & Wahrehouse \#05 & $\# 10503$ \\
\hline 6 & P22 & 1 & 5 & Wahrehouse \#05 & $\# 10501$ \\
\hline 7 & Blocuri_U & 1 & 5 & Wahrehouse \#05 & $\# 10501$ \\
\hline 8 & R1 & 1 & 10 & Wahrehouse \#05 & $\# 10503$ \\
\hline 9 & Belvedere & 1 & 5 & Wahrehouse \#05 & $\# 10501$ \\
\hline 10 & R3 & 1 & 14 & Wahrehouse \#05 & $\# 10505$ \\
\hline 11 & ComplexLeu & 1 & 10 & Wahrehouse \#05 & $\# 10503$ \\
\hline 12 & CantinaLeu & 1 & 5 & Wahrehouse \#05 & $\# 10501$ \\
\hline 13 & Grozavesti1 & 1 & 10 & Wahrehouse \#05 & $\# 10503$ \\
\hline 14 & Grozavesti2 & 1 & 10 & Wahrehouse \#05 & $\# 10503$ \\
\hline 15 & ANEFS & 1 & 12 & Wahrehouse \#03 & $\# 10304$ \\
\hline 16 & Drept & 1 & 12 & Wahrehouse \#03 & $\# 10304$ \\
\hline 17 & CamineConstructii & 1 & 7 & Wahrehouse \#03 & $\# 10302$ \\
\hline
\end{tabular}

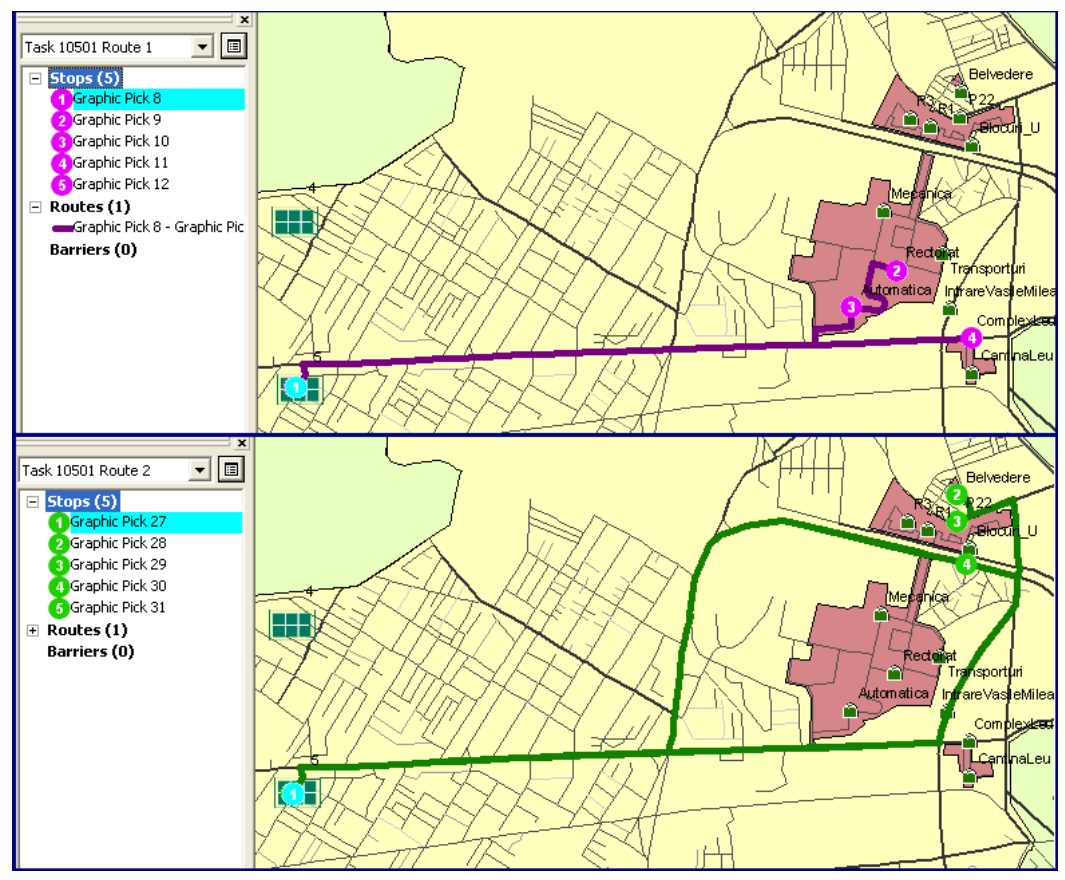

Figure 7: Route layers. 
Each collecting point needs 12 minutes for loading/unloading the container. The total travel time along all routes is calculated based on each arc travel time from the network dataset. Detailed schedule table are obtained for each collecting vehicle.

\section{Conclusion}

Selective waste collecting in university campuses is important for minimizing the impact on the environment. The collection services have to be organised in campuses with restricted access and in traffic congested urban areas. In order to reduce the total costs, the vehicle fuel consumption and pollutant emission, efficient methods for planning the recyclable waste collecting trips are necessary.

GIS tools and procedure are useful for optimal planning of selective waste collection. The GIS model presented in this paper solves the routing problem. Additional programming algorithm is necessary for planning the collection services. The developed methods provide informational and technological support for public authorities and selective waste collection operators. Decision makers can also use the model to explore tradeoffs between planning alternatives and identify the optimal solution. The proposed model could be easily adapted for other practical special cases.

\section{Acknowledgement}

The paper has been supported by the research contract 92110 "Civic Education for Selective Waste Collection in University Campuses - ECOSED”, funding by UEFISCDI/PNCDI-II.

\section{References}

[1] Bodin, L.D., Twenty Years of Routing and Scheduling, Operations Research, 38, pp. 571-579, 1990.

[2] Martelo, S., Laporte, G., Minoux, M., Ribeiro, C. (eds). Survey in combinatorial optimization, Volume 31 of Annals of Discrete Mathematics, North-Holland, Amsterdam, 1987.

[3] ECOROM, ECOROM Annual Report, Bucuresti, Romania, 2011.

[4] ESRI, ArcGIS9. Network Analyst, Redlands, USA, 2005. 